\title{
Functional magnetic resonance imaging evaluation of lumbosacral radiculopathic pain
}

\author{
Alex J. Koefman, MBBS, ${ }^{1}$ Melissa Licari, PhD, ${ }^{2}$ Michael Bynevelt, FRANZCR, ${ }^{3}$ and \\ Christopher R. P. Lind, FRACS ${ }^{1,4}$
}

Departments of ${ }^{1}$ Neurosurgery and ${ }^{3}$ Neuroradiology, Sir Charles Gairdner Hospital; and Schools of ${ }^{2}$ Sport Science, Exercise \& Health and ${ }^{4}$ Surgery, University of Western Australia, Perth, Western Australia, Australia

\begin{abstract}
OBJECTIVE An objective biomarker for pain is yet to be established. Functional MRI (fMRI) is a promising neuroimaging technique that may reveal an objective radiological biomarker. The purpose of this study was to evaluate fMRI technology in the setting of lumbosacral radiculopathy and discuss its application in revealing a biomarker for pain in the future.
\end{abstract}

METHODS A prospective, within-participant control study was conducted. Twenty participants with painful lumbosacral radiculopathy from intervertebral disc pathology were recruited. Functional imaging of the brain was performed during a randomly generated series of nonprovocative and provocative straight leg raise maneuvers.

RESULTS With a statistical threshold set at $p<0.000001,3$ areas showed significant blood oxygen level-dependent (BOLD) signal change: right superior frontal gyrus $(x=2, y=13, z=48, k=29$, Brodmann area 6 [BA6]), left supramarginal cortex $(x=-37, y=-44, z=33, k=1084$, BA40), and left parietal cortex $(x=-19, y=-41, z=63, k=354$, BA5). With a statistical threshold set at $p<0.0002,2$ structures showed significant BOLD signal change: right putamen $(x=29$, $y=-11, z=6, k=72$ ) and bilateral thalami (right: $x=23, y=-11, z=21, k=29 ; x=8, y=-11, z=9, k=274$; and left: $x=$ $-28, y=-32, z=6, k=21)$.

CONCLUSIONS The results in this study compare with those in previous studies and suggest that fMRI technology can provide an objective assessment of the pain experience.

http://thejns.org/doi/abs/10.3171/2016.3.SPINE151230

KEY WORDS functional MRI; pain; radiculopathy, technique

$\mathrm{T}$ HE assessment and management of patients with back pain are often made difficult due to inherent temporal and psychosocial factors that confound the experience and therefore the reporting of pain. An objective biomarker for pain, as an adjunct to current clinical and radiological assessment tools, would be particularly useful in this setting. Functional MRI (fMRI) is a promising neuroimaging technique that can be used to objectively reveal a radiological biomarker in the future. Historically, fMRI became possible when, in 1990, investigators observed in studies of the murine brain that the characteristics of MR images were dependent on the degree of oxygenation and varied with the functional state. ${ }^{20}$ Human application quickly followed in 1991 when Belliveau et al. ${ }^{3}$ first described mapping of the human visual cortex utilizing fMRI.
Since then, significant progress has been made in the use of fMRI paradigms to interrogate brain activity. It has emerged that 6 core cerebral substrates are frequently associated with the pain state: primary and secondary somatosensory cortices (S1 and S2, respectively), insular cortex, thalamus, anterior cingulate cortex (ACC), and prefrontal cortex (PFC). Numerous studies have demonstrated that these regions of the brain show different fMRI activation patterns during different pain states, including migraine, ${ }^{23}$ fibromyalgia syndrome, and complex regional pain syndrome,${ }^{8}$ suggesting that fMRI signatures may be unique for particular pain states. Furthermore, Schweinhardt et al. ${ }^{24}$ highlighted the importance of the insular cortex in those suffering from neuropathic pain by demonstrating that the magnitude of activation in the caudal anterior insular region paralleled the perceived intensity of allodynic pain.

ABBREVIATIONS ACC = anterior cingulate cortex; BA = Brodmann area; BOLD = blood oxygen level-dependent; fMRI = functional MRI; LSR = lumbosacral radiculopathy; $\mathrm{mPFC}=$ medial PFC; ODI = Oswestry Disability Index; PFC = prefrontal cortex; S1 = primary somatosensory cortex; $\mathrm{S} 2$ = secondary somatosensory cortex; SLR = straight leg raise; VAS = visual analog scale. 
As yet, very few functional neuroimaging studies have been undertaken to examine the experience of back pain. In participants suffering chronic low-back pain, Kobayashi et al..$^{14}$ noted augmented activation in the right insular, supplementary motor, and posterior cingulate cortices compared with that in healthy controls. Furthermore, Seminowicz et al. ${ }^{25}$ highlighted cortical activation disparities in participants with back pain before and after symptomatic treatment. Additionally, patterns of altered periaqueductal gray functional connectivity in participants suffering chronic low-back pain have been demonstrated using fMRI by both Yu et al..$^{33}$ and Kong et al. ${ }^{16}$ It has been shown that concomitant patient psychosocial factors affect blood oxygen level-dependent (BOLD) signal responses within the normal pain matrix. This was illustrated in a discussion by Wand et al., ${ }^{30}$ who reviewed work in this area by both Flor et al. ${ }^{11}$ and Lloyd et al. ${ }^{17}$ Flor and colleagues initially demonstrated that representation of the lower back in S1 was shifted medially and expanded in participants with low-back pain and that the extent of expansion was closely associated with pain chronicity. Lloyd and associates showed similar findings in participants with low-back pain, but only in those who were distressed. Wand et al. proposed that the S1 shifts were a feature of emotional impact rather than the low-back pain itself.

The purpose of this study was to evaluate the ability of fMRI to map the experience of neuropathic pain in cortical and subcortical regions of the brain. Importantly, we postulate that, by applying fMRI in the setting of lumbosacral radiculopathy (LSR), the significant psychosocial and chronicity-related confounders that may influence fMRI results, as suggested by Wand et al., ${ }^{30}$ will be largely eliminated. Furthermore, Schweinhardt et al. ${ }^{24}$ showed a functional segregation of the anterior insular cortex that was dependent on whether the pain was neuropathic in origin or an experimental noxious stimulus, suggesting that the processing of clinical pain in the brain differs from that of experimental pain. With this in mind, we elected to use provocative straight leg raise (SLR) to elicit neuropathic pain during fMRI data acquisition rather than the experimental cutaneous noxious stimuli methods employed by most previously performed fMRI studies. Sharma et al. ${ }^{26}$ have described and validated provocative SLR for use during fMRI data acquisition.

\section{Methods \\ Participants}

The Sir Charles Gairdner Group Human Research Ethics Committee granted study approval. Twenty participants who had no preexisting chronic pain or neurological or psychiatric conditions were recruited. There were 10 males and 10 females with a combined mean age of 40.4 years. To be eligible for the study, participants were required to demonstrate reliable exacerbation of radiculopathy on provocative SLR and adequate resolution of radiculopathy when the affected lower limb was returned to the resting position. Participants were eligible for study inclusion if the severity of their pain on provocation was $7 / 10$ or greater according to the visual analog scale (VAS). Participants were assessed in an outpatient clinic setting and completed an Oswestry Disability Index (ODI) questionnaire ${ }^{10}$ at the time of enrollment.

\section{Study Design}

Our study was designed as a prospective, within-subject control study. Participants underwent fMRI of the brain before proceeding to surgery. The surgical technique was limited to microdiscectomy using microsurgical techniques and an operating microscope and was performed by a single experienced consultant neurosurgeon (C.R.P.L.). Participants were reassessed between 1 and 3 months (mean 1.4 months) for residual radiculopathy and postoperative complications and repeat ODI. One patient reported the onset of mild recurrent symptoms 2 weeks after surgery, and 1 patient reported new symptoms on the contralateral side. Two patients reported new-onset back pain following surgery, but it gradually resolved. Postoperative ODI scores ranged from 0 to 5 at the time of scheduled follow-ups. Participants were instructed to continue their analgesia for the duration of the study, and while the literature reports that the effect of medication may influence fMRI data, ${ }^{31}$ it was not feasible for several of the participants to cease their medication given the severity of their symptoms. Thus, it was decided that all participants would continue their current medications.

\section{Functional MRI Pain Paradigm}

The fMRI paradigm was a block design. There were 3 conditions: "rest," in which each participant's affected lower limb was lying on the MRI bench with an author's hand under the ankle to control for BOLD activations due to tactile stimulation; "partial" SLR, in which the affected lower limb was passively raised $5 \mathrm{~cm}$ off the MRI bench at the hip joint with the knee fully extended; and "painful" SLR, in which the affected lower limb was passively raised by the chief investigator to a point pre-identified as eliciting an exacerbation of the radiculopathy with a severity of at least $7 / 10$ according to the VAS. Both the partial and the painful SLR conditions lasted for a 30-second period and were interspersed with 12 seconds of rest. The block design included a randomly generated order of partial and painful SLR to control for subject expectation, which is known to alter the pain experience,,$^{15}$ and this strategy is paralleled in radiological studies of pain. Hsieh et al. ${ }^{12}$ reported an increase in regional blood flow in the PFC, caudal ACC, and periaqueductal gray in participants anticipating a painful event. ${ }^{17}$

\section{Image Acquisition, Preprocessing, and Analysis}

All MRI examinations were performed using a 3-T Philips Achieva TX scanner with participants wearing a 12-channel head coil. High-resolution anatomical images were acquired first (T1-weighted 3D fast field echo, 175 slices, $1 \times 1 \times 1 \mathrm{~mm}$ ) followed by 2 functional runs (T2weighted gradient echo, TR $300 \mathrm{msec}$, TE $35 \mathrm{msec}$, flip angle $90^{\circ}, 36$ axial slices with a thickness of $3 \mathrm{~mm}$, interslice gap $0 \mathrm{~mm}$ ).

Magnetic resonance imaging data were analyzed using Brain Voyager QX software (version 2.8, Brain Innovation). Preprocessing of the data included both $3 \mathrm{D}$ motion 
correction and temporal filtering to remove head movement and signal drift, respectively. Four participants were removed at this stage of the study as they exhibited more than $1 \mathrm{~mm}$ of head motion. All images were smoothed using an 8-mm full width at half maximum Gaussian kernel to improve registration across participants. Each participant's functional images were coregistered with their high-resolution anatomical image and were normalized to the Talairach space. Contrast maps were created for each participant. In a second-level multisubject random effects model (general linear model [GLM]), whole brain analysis was performed to explore differences in right- and left-limb radiculopathy participants. No differences were found, so participant data were collapsed and a series of whole brain analyses were performed to examine cortical areas activated between conditions at corrected ( $\mathrm{p}<$ $0.000001)$ and uncorrected levels ( $\mathrm{p}<0.0002)$.

\section{Results}

\section{Clinical Characteristics of Participants}

The majority of the disc pathology was at L4 -5 giving rise to L-5 radiculopathy, with 1 far lateral disc protrusion at this level causing an L-4 radiculopathy (Table 1). Of the 20 participants recruited, 16 had head motion $<1 \mathrm{~mm}$ during fMRI data acquisition and thus were included in the statistical analysis. Of those 16 subjects, 9 had left-sided radiculopathy and 7 had right-sided radiculopathy.

\section{Whole Brain Analysis: Condition Comparison}

Painful and partial (nonpainful) leg raise were compared with the rest condition. There were 3 significant fMRI BOLD signal changes detected in the painful SLR

\section{TABLE 1. Details of study participants}

\begin{tabular}{lc}
\hline \multicolumn{1}{c}{ Parameter } & Value \\
\hline Sex & \\
M & 10 \\
F & 10 \\
\hline Mean age in yrs (range) & $40.35(19-72)$ \\
\hline Level of disc pathology & 13 \\
L4-5 & 7 \\
L5-S1 & \\
\hline Nerve root involvement & 14 \\
L-5 & 6 \\
S-1 & 10 \\
\hline Side of radiculopathy & 10 \\
Rt & $7.5(3-15)$ \\
Lt & $8(7-10)$ \\
\hline Mean duration of radiculopathy in mos (range) & 50 \\
\hline Mean VAS score (range) & 45 \\
\hline Cause of radiculopathy (\%) & 5 \\
Disc protrusion & $17-28$ \\
Disc extrusion & $0-5$ \\
Sequestered disc fragment & \\
\hline ODI score (range) & \\
Preop & \\
Postop & \\
\hline
\end{tabular}

versus rest analysis (Table 2 and Fig. 1). These were in the right superior frontal gyrus, left superior parietal lobe, and left supramarginal gyrus. No differences were found at a corrected level between the partial versus rest and the painful versus partial conditions.

When examining the data at a less conservative level ( $p<0.0002$ ), 3 areas of activation differences were found between the painful and partial SLR conditions (Table 3 and Fig. 2). Bilateral thalami showed BOLD activation, with the largest cluster of activation difference seen on the right. The third area to show significant BOLD signal change was the right putamen.

\section{Discussion}

Of the 6 previously identified core substrates of pain, 2 showed significant BOLD activation changes in the present study, namely the thalamus and the PFC. In addition, there were significant BOLD activation differences in the parietal lobe and the putamen. To our knowledge, this is the first fMRI study to examine radiculopathic pain as a means to reduce the confounding effects of temporal and psychosocial factors on fMRI evaluation of the cerebral substrates of pain.

Corrected data analysis in the present study (Bonferroni correction $p<0.000001$ ) revealed 3 areas of significance in BOLD signal changes between the rest and pain states. The first of these areas was the right superior frontal gyrus $(\mathrm{x}=2, \mathrm{y}=13, \mathrm{z}=48, \mathrm{k}=29$, Brodmann area 6 [BA6]). The frontal cortex is large and has a complex role in pain processing. It is often divided into the medial prefrontal area, dorsal prefrontal area, and dorsolateral prefrontal area. It is thought that the medial PFC (mPFC) is more closely related to the emotional aspect of pain (the medial pain matrix also involving the limbic system), while the lateral and dorsal PFCs are more associated with the cognitive aspects of pain. Our results are consistent with those of Baliki et al., ${ }^{2}$ who showed that patients with back pain have increased activity in the MPFC during episodes of sustained pain and that the mPFC activity is strongly related to the intensity of back pain. The frontal cortex is also thought to be important in modulating pain via corticosubcortical and corticocortical pathways. ${ }^{1,6}$ Evidence of such modulation could be an important component of a neuroimaging pain biomarker. The BOLD signal change in BA6 in the present study is in keeping with the study by Coghill et al. ${ }^{8}$ In their study, $\mathrm{H}_{2}{ }^{15} \mathrm{O}$ PET of cerebral blood flow was used to map the brain activation induced by thermal stimulation. Their results showed that both BA6 and

TABLE 2. Whole brain analysis: condition comparison of painful SLR and rest*

\begin{tabular}{|c|c|c|c|c|c|}
\hline \multirow[b]{2}{*}{ Anatomical Region } & \multirow{2}{*}{$\begin{array}{l}\text { Cluster } \\
\text { (k) }\end{array}$} & \multicolumn{3}{|c|}{$\begin{array}{c}\text { Peak Talairach } \\
\text { Coordinates }\end{array}$} & \multirow[b]{2}{*}{ BA } \\
\hline & & $x$ & y & z & \\
\hline Rt superior frontal gyrus & 29 & 2 & 13 & 48 & 6 \\
\hline Lt superior parietal lobe & 354 & -19 & -41 & 63 & 5 \\
\hline Lt supramarginal gyrus & 1084 & -37 & -44 & 33 & 40 \\
\hline
\end{tabular}

* Bonferroni corrected, $p<0.000001$. 

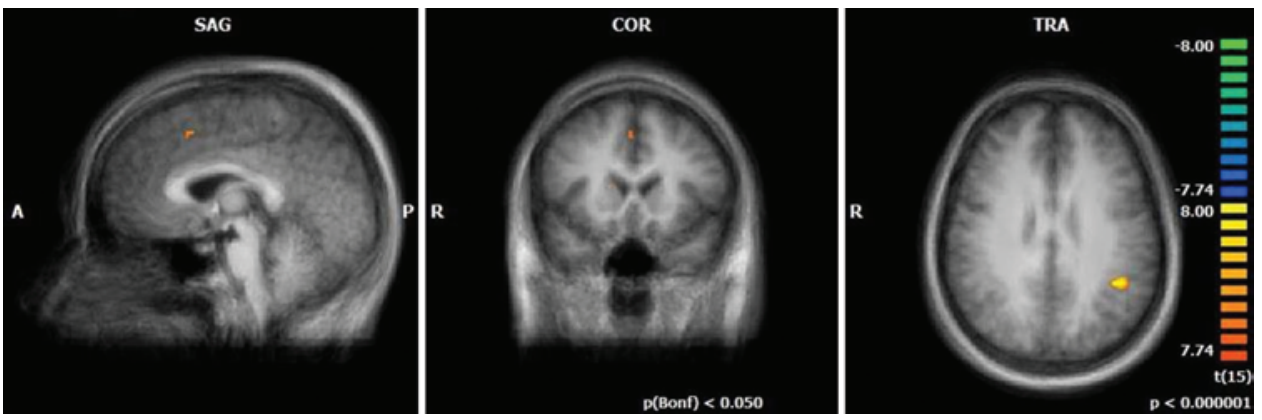

FIG. 1. Main effect of painful SLR compared with the rest condition (Bonferroni [Bonf] corrected, $p<0.000001$ ) showing significant BOLD signal change in the right superior frontal gyrus in the sagittal (SAG) and coronal (COR) sections and in the left supramarginal gyrus in the axial (TRA) section.

BA40 (the second region to show significant BOLD signal change in the present study) were intensity independent and therefore likely to be important substrates in the processing of pain. Brodmann area $40(\mathrm{x}=-37, \mathrm{y}=-44, \mathrm{z}$ = 33) showed the largest cluster $(\mathrm{k}=1084)$ in the present study. It is known that the dominant (often the left) supramarginal cortex is important for receptive language function; thus, it is possible that activation here reflects the participant's cognitive appreciation of the pain, while the mPFC activation reflects their emotional response to the pain. Therefore, it is unlikely that BA40 and medial BA6 would contribute significantly to the characterization of a potential biomarker of pain.

The third area was the left parietal cortex $(\mathrm{x}=-19, \mathrm{y}$ $=-41, \mathrm{z}=63, \mathrm{BA} 5)$. The $\mathrm{S} 1$ and $\mathrm{S} 2$ are recognized substrates of central pain processing, though the exact roles of each area are the subject of ongoing investigation. Electroencephalography mapping studies show that the earliest pain-induced brain activity originates in the vicinity of the S2. In contrast, tactile stimuli activate this region only after processing in the S1, which supports the theory derived from anatomical studies that the $\mathrm{S} 2$ region is a primary receiving area for nociceptive input to the brain. ${ }^{1}$ Furthermore, Strigo et al. ${ }^{27}$ compared cortical activations from esophageal distension and contact heat stimuli on the chest. For these subjects, the visceral and cutaneous pain both led to activations in S2, but the exact loci within the regions differed for the 2 types of pain, thus supporting the idea that there may be subregional differences in the processing of different types of pain.

At the less conservative uncorrected level in the present study, 2 activation differences were seen between partial and pain states. The first of these areas was the right

TABLE 3. Whole brain analysis: condition comparison of painful SLR and partial SLR*

\begin{tabular}{|c|c|c|c|c|}
\hline \multirow{2}{*}{$\begin{array}{c}\text { Anatomical } \\
\text { Region }\end{array}$} & \multirow[b]{2}{*}{ Cluster (k) } & \multicolumn{3}{|c|}{ Peak Talairach Coordinates } \\
\hline & & $x$ & $\mathrm{y}$ & z \\
\hline Rt putmen & 72 & 29 & -11 & 6 \\
\hline \multirow[t]{2}{*}{ Rt thalamus } & 29 & 23 & -11 & 21 \\
\hline & 274 & 8 & -11 & 9 \\
\hline Lt thalamus & 21 & -28 & -32 & 6 \\
\hline
\end{tabular}

* Uncorrected, $p<0.0002$. putamen $(\mathrm{x}=29, \mathrm{y}=-11, \mathrm{z}=6, \mathrm{k}=72)$. Current understanding of alterations in cortical and subcortical regions in pain suggests that the basal ganglia are uniquely involved in thalamo-cortico-basal ganglia loops to integrate many aspects of pain. ${ }^{5}$ These include the integration of motor, emotional, autonomic, and cognitive responses to pain. Electrophysiological data show that the basal ganglia receive nociceptive and nonnoxious somatosensory information. Interestingly and somewhat unsurprisingly given the close functional relationship of the basal ganglia to the motor system, the primary role of the putamen probably relates to immediate defense and withdrawal behavior. ${ }^{4}$ It was observed during painful leg raise that the participants would flinch-an observation that will be part of the explanation for putamen activation in the present study. Though impossible to prove, it is also likely that participants engaged in some degree of avoidance behavior planning during the "no pain" state. Evidence suggests that the putamen also encodes stimulus intensity ${ }^{7}$ and may therefore be a useful marker in the future in the context of patients who demonstrate a discrepancy between clinical symptoms and radiological findings.

The second area that trended toward significance was the thalamus. Bilateral thalami showed trends toward significant activation change, the right more so than the left (largest cluster on the right $\mathrm{k}=274$ compared with $\mathrm{k}=$ 21 on the left), and demonstrating 2 areas of BOLD signal change $(\mathrm{x}=23, \mathrm{y}=-11, \mathrm{z}=21$ and $\mathrm{x}=8, \mathrm{y}=-11, \mathrm{z}$ $=9$ ). This was not surprising, as the thalamus is a wellestablished integral substrate of pain processing. Interest-
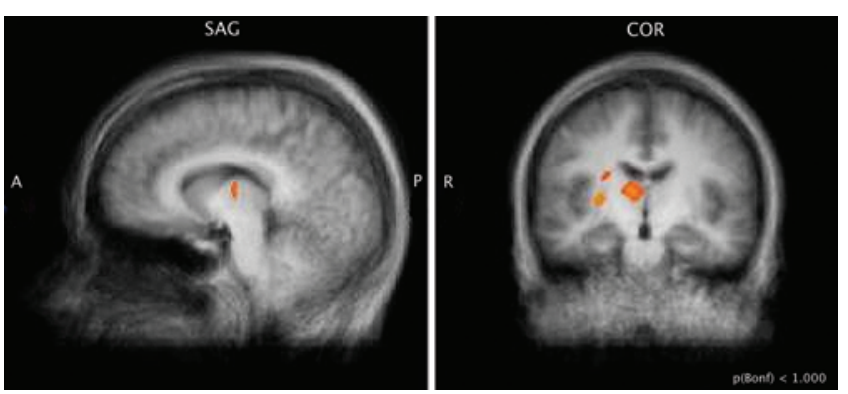

FIG. 2. Main effect of painful SLR compared with partial SLR (uncorrected, $p<0.0002$ ) showing significant BOLD signal change in the right thalamus and putamen. 
ingly, BOLD signal changes in the thalamus during painful stimulus have been reported as highly variable. ${ }^{28}$ This variability may be partly dependent on the chronicity of the pain stimulus. A SPECT blood flow study by Ushida et al. ${ }^{29}$ showed a strong relationship between the time of onset of complex regional pain syndrome symptoms and thalamic activity. Patients with symptoms for only 3-7 months demonstrated thalamic hyperperfusion, and patients with longer-term symptoms (24-36 months) showed hypoperfusion. This finding is consistent with results of the present study in which all participants had LSR for less than 24 months. Furthermore, thalamic atrophy has been reported in patients with chronic back pain. ${ }^{28}$ Thus, the increased BOLD signal of the thalami in the present study may not only reflect integral central processing of pain or but perhaps also arousal to pain (during painful leg raise) - a concept hypothesized by Peyron et al. ${ }^{21}$

Despite the insular and anterior cingulate cortices being recognized substrates of cerebral pain processing, neither showed significant BOLD signal changes in the present study. The posterior insular cortex seems to be more related to sensory aspects of pain, while the anterior parts are associated with emotional, cognitive, and memory-related aspects of pain..$^{1,18}$ The ACC is thought to serve as an integrative processing domain related to several cognitive and emotional experiences. ${ }^{18,19}$ The absence of significant BOLD signal change in these 2 areas in the present study may be attributable to the neuropathic nature of LSR. It has been previously reported that the "pain matrix" involved in processing noxious pain differs from that which processes neuropathic pain. In a study by Ducruex et al., ${ }^{9}$ the PFC was the only area consistently activated by pathological evoked pain in participants with syringomyelia (brush-evoked allodynia). Similarly, in another study in participants with syringomyelia, Petrovic et al. ${ }^{20}$ showed no significant activation in the insular cortex in patients with allodynia. Not all functional neuroimaging studies of neuropathic pain are consistent with these results, however. Witting et al. ${ }^{32}$ studied 9 patients with traumatic nerve injury and found that a significant increase in regional cerebral blood flow was observed bilaterally in S2, in the ipsilateral anterior insular cortex, contralateral orbitofrontal cortex (BA11), and cerebellum, but not in the ACC.

There are several limitations to the present study. First, despite a population size comparable to those in similar studies, our overall sample size was small. Large-scale studies would be required if a robust and reproducible biomarker were to be tested. Second, no significant BOLD signal change was observed between the painful and partial SLR at a corrected level. The partial SLR was designed to control for the somatosensory aspects of the SLR. In reality, it is likely that even the partial SLR, in which the lower limb was passively raised $5 \mathrm{~cm}$ from the bed, was still somewhat provocative. When the statistical threshold was lowered to a less conservative uncorrected level, differences were observed between painful and partial SLR, which appear to be clinically significant. Third is the fMRI technology itself. One major criticism of the technology relates to data interpretation rather than data acquisition. It is yet to be determined what the exact relationship between the BOLD signal change (fMRI visible local area of "activation") and the pain experience is. As Jardetzky ${ }^{13}$ demonstrates, the local "activated" area may represent a command or collection center that receives or sends signals to many points in the peripheral brain where they are unobservable because of the low sensitivity of fMRI. Alternatively, the local activated region may be a completely self-contained center for the experience of pain.

\section{Conclusions}

Upon concluding our study, it seems reasonable to suggest that fMRI could form the basis of an objective biomarker for pain in the future. The technology appears to have the capability to identify one or more core cerebral substrates repeatedly, to potentially distinguish neuropathic from non-neuropathic pain, and to give an indication of pain intensity. The purpose of this study was to investigate the cerebral substrates of clinical pain with a control in place (LSR as a study template) for temporal and psychosocial factors. Further research is required to better delineate the effects of these factors on fMRI data and on the normal cerebral patterns of pain processing.

\section{Acknowledgments}

We gratefully acknowledge the contributions of the radiography staff at the Department of Neuroradiology, Sir Charles Gairdner Hospital, Perth, Australia.

\section{References}

1. Apkarian AV, Bushnell MC, Treede RD, Zubieta JK: Human brain mechanisms of pain perception and regulation in health and disease. Eur J Pain 9:463-484, 2005

2. Baliki MN, Chialvo DR, Geha PY, Levy RM, Harden RN, Parrish TB, et al: Chronic pain and the emotional brain: specific brain activity associated with spontaneous fluctuations of intensity of chronic back pain. J Neurosci 26:1216512173, 2006

3. Belliveau JW, Kennedy DN Jr, McKinstry RC, Buchbinder BR, Weisskoff RM, Cohen MS, et al: Functional mapping of the human visual cortex by magnetic resonance imaging. Science 254:716-719, 1991

4. Bingel U, Gläscher J, Weiller C, Büchel C: Somatotopic representation of nociceptive information in the putamen: an event-related fMRI study. Cereb Cortex 14:1340-1345, 2004

5. Borsook D, Upadhyay J, Chudler EH, Becerra L: A key role of the basal ganglia in pain and analgesia-insights gained through human functional imaging. Mol Pain 6:27, 2010

6. Burgmer M, Pogatzki-Zahn E, Gaubitz M, Stüber C, Wessoleck E, Heuft G, et al: Fibromyalgia unique temporal brain activation during experimental pain: a controlled fMRI Study. J Neural Transm (Vienna) 117:123-131, 2010

7. Chudler EH, Dong WK: The role of the basal ganglia in nociception and pain. Pain 60:3-38, 1995

8. Coghill RC, Gilron I, Iadarola MJ: Hemispheric lateralization of somatosensory processing. J Neurophysiol 85:26022612,2001

9. Ducreux D, Attal N, Parker F, Bouhassira D: Mechanisms of central neuropathic pain: a combined psychophysical and fMRI study in syringomyelia. Brain 129:963-976, 2006

10. Fairbank JC, Pynsent PB: The Oswestry Disability Index. Spine (Phila Pa 1976) 25:2940-2952, 2000

11. Flor H, Braun C, Elbert T, Birbaumer N: Extensive reorgani- 
zation of primary somatosensory cortex in chronic back pain patients. Neurosci Lett 224:5-8, 1997

12. Hsieh JC, Stone-Elander S, Ingvar M: Anticipatory coping of pain expressed in the human anterior cingulate cortex: a positron emission tomography study. Neurosci Lett 262:61-64, 1999

13. Jardetzky O: FMRI in brain research in its historical context. Am J Bioeth 8:43-45, 2008

14. Kobayashi Y, Kurata J, Sekiguchi M, Kokubun M, Akaishizawa T, Chiba Y, et al: Augmented cerebral activation by lumbar mechanical stimulus in chronic low back pain patients: an FMRI study. Spine (Phila Pa 1976) 34:2431-2436, 2009

15. Kong J, Kaptchuk TJ, Polich G, Kirsch I, Vangel M, Zyloney $\mathrm{C}$, et al: An fMRI study on the interaction and dissociation between expectation of pain relief and acupuncture treatment. Neuroimage 47:1066-1076, 2009

16. Kong J, Spaeth RB, Wey HY, Cheetham A, Cook AH, Jensen $\mathrm{K}$, et al: $\mathrm{S} 1$ is associated with chronic low back pain: a functional and structural MRI study. Mol Pain 9:43, 2013

17. Lloyd D, Findlay G, Roberts N, Nurmikko T: Differences in low back pain behavior are reflected in the cerebral response to tactile stimulation of the lower back. Spine (Phila Pa 1976) 33:1372-1377, 2008

18. Meier ML, Brügger M, Ettlin DA, Luechinger R, Barlow A, Jäncke L, et al: Brain activation induced by dentine hypersensitivity pain-an fMRI study. J Clin Periodontol 39:441-447, 2012

19. Ogawa S, Lee TM, Kay AR, Tank DW: Brain magnetic resonance imaging with contrast dependent on blood oxygenation. Proc Natl Acad Sci U S A 87:9868-9872, 1990

20. Petrovic P, Ingvar M, Stone-Elander S, Petersson KM, Hansson P: A PET activation study of dynamic mechanical allodynia in patients with mononeuropathy. Pain 83:459-470, 1999

21. Peyron R, Laurent B, García-Larrea L: Functional imaging of brain responses to pain. A review and meta-analysis (2000). Neurophysiol Clin 30:263-288, 2000

22. Ploghaus A, Tracey I, Gati JS, Clare S, Menon RS, Matthews PM, et al: Dissociating pain from its anticipation in the human brain. Science 284:1979-1981, 1999

23. Scheef L, Jankowski J, Daamen M, Weyer G, Klingenberg M, Renner J, et al: An fMRI study on the acute effects of exercise on pain processing in trained athletes. Pain 153:17021714,2012

24. Schweinhardt P, Glynn C, Brooks J, McQuay H, Jack T, Chessell I, et al: An fMRI study of cerebral processing of brush-evoked allodynia in neuropathic pain patients. Neuroimage 32:256-265, 2006

25. Seminowicz DA, Wideman TH, Naso L, Hatami-Khoroushahi Z, Fallatah S, Ware MA, et al: Effective treatment of chronic low back pain in humans reverses abnormal brain anatomy and function. J Neurosci 31:7540-7550, 2011

26. Sharma HA, Gupta R, Olivero W: fMRI in patients with lumbar disc disease: a paradigm to study patients over time. J Pain Res 4:401-405, 2011
27. Strigo IA, Duncan GH, Boivin M, Bushnell MC: Differentiation of visceral and cutaneous pain in the human brain. $\mathbf{J}$ Neurophysiol 89:3294-3303, 2003

28. Taylor KS, Davis KD: Stability of tactile- and pain-related fMRI brain activations: an examination of threshold-dependent and threshold-independent methods. Hum Brain Mapp 30:1947-1962, 2009

29. Ushida T, Fukumoto M, Binti C, Ikemoto T, Taniguchi S, Ikeuchi M, et al: Alterations of contralateral thalamic perfusion in neuropathic pain. Open Neuroimaging J 4:182-186, 2010

30. Wand BM, Parkitny L, O'Connell NE, Luomajoki H, McAuley JH, Thacker M, et al: Cortical changes in chronic low back pain: current state of the art and implications for clinical practice. Man Ther 16:15-20, 2011

31. Wang W, Zhang M, Wang Y, Jin C, Yan B, Ma S: 5-HT modulation of pain in SI and SII revealed by fMRI. Zhong Nan Da Xue Xue Bao Yi Xue Ban 35:185-193, 2010

32. Witting N, Kupers RC, Svensson P, Jensen TS: A PET activation study of brush-evoked allodynia in patients with nerve injury pain. Pain 120:145-154, 2006

33. Yu R, Gollub RL, Spaeth R, Napadow V, Wasan A, Kong J: Disrupted functional connectivity of the periaqueductal gray in chronic low back pain. Neuroimage Clin 6:100-108, 2014

\section{Disclosures}

The authors report no conflict of interest concerning the materials or methods used in this study or the findings specified in this paper.

\section{Author Contributions}

Conception and design: Koefman, Lind. Acquisition of data: Koefman, Licari. Analysis and interpretation of data: Koefman, Licari. Drafting the article: Koefman, Lind. Critically revising the article: Koefman, Licari, Lind. Reviewed submitted version of manuscript: all authors. Approved the final version of the manuscript on behalf of all authors: Koefman. Statistical analysis: Koefman, Licari. Administrative/technical/material support: Koefman, Bynevelt. Study supervision: Koefman.

\section{Supplemental Information}

\section{Previous Presentations}

The results of this research were presented at the 2015 Neurosurgical Society of Australasia Annual Scientific Meeting held in Auckland, New Zealand, on September 30-October 2, 2015.

\section{Correspondence}

Alex J. Koefman, Department of Neurosurgery, Royal Brisbane Hospital, Butterfield St., Henderson, Brisbane, QLD 4006, Australia.email: akoefman@gmail.com. 